\title{
Quantifying success and attempts on auto-graded homework when using an interactive textbook
}

\section{Prof. Matthew W Liberatore, The University of Toledo}

Matthew W. Liberatore is a Professor of Chemical Engineering at the University of Toledo. He earned a B.S. degree from the University of Illinois at Chicago and M.S. and Ph.D. degrees from the University of Illinois at Urbana-Champaign, all in chemical engineering. His current research involves the rheology of complex fluids as well as active learning, reverse engineering online videos, and interactive textbooks. His website is: http://www.utoledo.edu/engineering/chemical-engineering/liberatore/

\section{Megan Davidson, The University of Toledo}

Kayla Chapman,

Kayla Chapman is currently studying chemical engineering at the University of Toledo and expects to earn a B.S. degree in 2021. She has assisted with multiple areas of research and data analysis regarding zyBooks reading participation and challenge activities. She became interested in performing research after completing a chemical engineering course that used zyBooks. 


\title{
Quantifying success and attempts on auto-graded homework when using an interactive textbook
}

\begin{abstract}
Online homework and interactive textbooks provide big data that can help answer many questions about student engagement and learning. In addition, some data is available to instructors in real time, so individual or class-level interventions do not need to wait for the next exam. In this study, a fully interactive online textbook, Material and Energy Balances zyBook, has been employed with several cohorts of students. Recent findings focused on quantify reading across three cohorts representing over 280 students and almost 300,000 reading interactions. Median reading rates were as high as $99 \%$ for over 1,300 interactions per student were found, but these reading clicks only quantify effort and not competence in the course material. Now, auto-graded homework questions with randomized numbers and content are explored. Students are allowed to attempt any question as many times as needed with each new attempt containing new numbers, content, or both. Three different, question-level metrics will be explored, namely percent correct, number of attempts before answering correctly, and total attempts. With over 500 auto-graded questions across 9 chapters, almost 200,000 student attempts are analyzed by chapter, question type, and cohort. Overall, students were successful on $88 \%$ of the questions; success varied by $10 \%$ between multiple choice, single numerical answer, and multiple numerical answer.
\end{abstract}

\section{Introduction}

Big data is encompassed by volume, velocity, and variety [1]. The rapid expansion of the Internet has led to big data in many ways. For example, the location, look, and size of buttons or ads on a screen has been thoroughly studied and optimized - in many cases to increase revenues [2]. In general, big data comes from interactivity, i.e., either a person clicking or scrolling on a webpage or sensors in either a home or chemical plant. In the same vein, combining interactivity with textbooks has begun to create big data in the engineering classroom.

Student-centered teaching techniques are commonly called active learning [3-8]. This type of pedagogy focuses on students learning by doing in many cases. Despite the large body of evidence supporting these best practices of teaching, adoption is not the norm. While not adopting the new standard techniques in laboratory research leaves faculty behind, the same expectation is not typical for many faculty's teaching. Changes in teaching techniques may be considered a social change, which generally changes more slowly than technology $[9,10]$. In this paper, the cross section of big data and active learning are studied in the context of an interactive textbook and online homework.

While textbook reading is not well quantified in higher education, or more specifically engineering education, interactive textbooks have contributed new information in recent years. Using clicks to quantify student's progress through animations and questions embedded within an interactive textbook, median reading rates significantly above $90 \%$ have been recorded. Consistently high reading rates over numerous cohorts (Figure 1) contrasts much lower reading rates for traditional textbooks, both at the due date and at the end of the semester [11-14]. Additionally, students earning A and B grades in a course demonstrated statistically significantly higher reading rates 
than C, D, and F students; Female students read statistically more than male students also. Overall, reading participation within an interactive textbook quantifies effort of students, while auto-graded questions provide more information related to each student's mastery of course material.

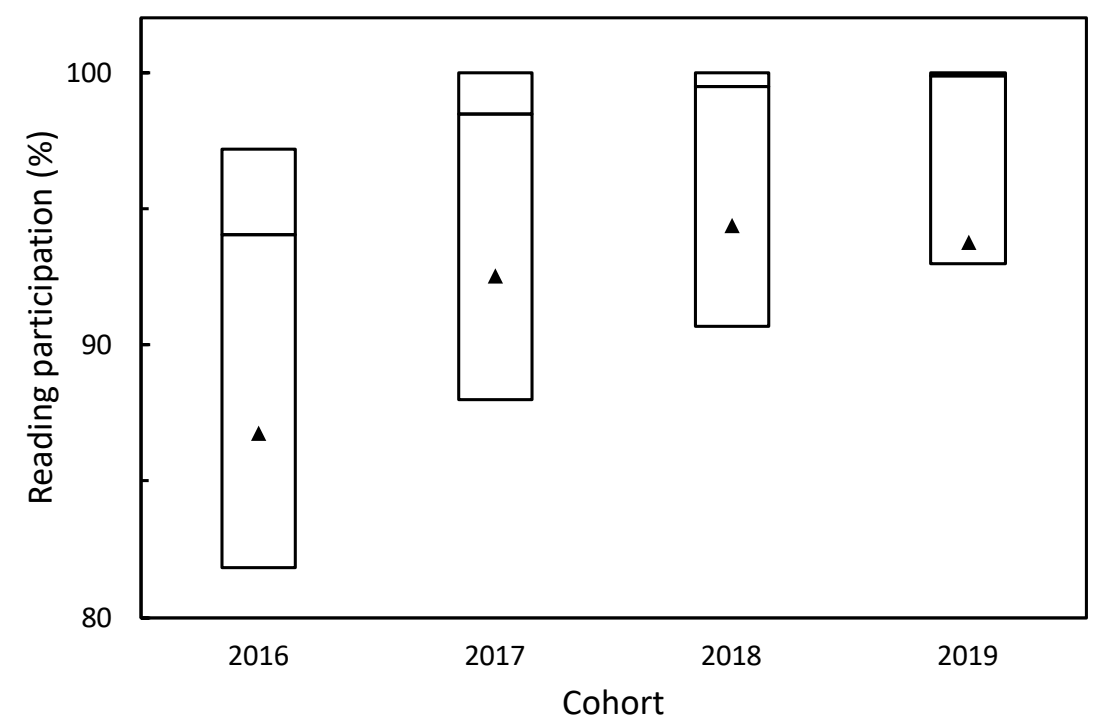

Figure 1. Reading participation for four cohorts of students using the Material and Energy Balances zyBook. Median, $1^{\text {st }}$ quartile, and $3^{\text {rd }}$ quartile are indicated by middle, bottom, and top of boxes, respectively. Triangles indicate mean. Details are available in other publications [11, 15], and 2019 data have not been previously published.

Many chemical engineering students experience several different online homework platforms in math, chemistry, and physics courses. Online homework questions can be directly connected to a textbook, provide additional features, or operate independently from course readings. Some formats limit attempts on each question, and others may deduct points with each incorrect response. Multiple attempts are generally observed as a productive learning strategy [16]. Feedback for incorrect responses may include full solutions, hints, or only the expected answeranswers could be numerical, drawn chemical structures, or text, such as names of chemical components. Some questions generate new numbers for each attempt, so students must rework the question before making another attempt. For example, a system called ALEKs requires multiple correct responses in a row for questions about one topic before the next topic is presented [17]. While each online homework system has pros and cons, providing immediate feedback to students is one significant advantage.

For more than a decade, significant findings related to online homework have been presented for engineering and technical courses $[18,19]$. For example, significant learning gains were observed in a statics course [20]. Also, a smaller percentage of a class earned failing grades when adopting online homework in the Material and Energy Balances course of interest here [21]. In general, the availability of multiple versions and attempts per question may help students feel more in control of their learning, which can be beneficial [22].

While the online homework results are primary in this paper, the course material also contributes. Material and Energy Balances (MEB) is nominally the first chemical engineering course, which 
introduces question solving and foundational topics, such as conservation of mass and energy. Many significant contributions can be found in the literature on best practices for teaching this course, including $[23,24]$. Here, the focus will be on student success and attempts related to online homework questions within an interactive textbook for MEB.

\section{Materials: Online homework within an interactive textbook}

The Material and Energy Balances zyBook is interactive content for use within any HTML5 compliant web browser without additional plug-ins. The content follows other textbooks for the course, which include over 80 sections, 140 animations, and 1300 clicks to read the whole book. Specifically of interest here are online homework questions, which are called challenge activities. Hundreds of challenge activities are available with the book, and more question levels have been added annually (Table 1).

Table 1. Online homework questions in MEB zyBook.

\begin{tabular}{|l|c|c|c|}
\hline Year & $\mathbf{2 0 1 7}$ & $\mathbf{2 0 1 8}$ & $\mathbf{2 0 1 9}$ \\
\hline $\begin{array}{l}\text { Auto-graded } \\
\text { challenge activity } \\
\text { questions (all) }\end{array}$ & 175 & 300 & 408 \\
\hline Multiple choice & 32 & 49 & 70 \\
\hline Single numeric & 116 & 217 & 263 \\
\hline Multiple numeric & 26 & 33 & 74 \\
\hline
\end{tabular}

The course consisted primarily of freshman students with enrollment between 88 and 105 students, approximately $60-65 \%$ male and $35-40 \%$ female at a public research university. The same instructor - one of the co-authors - led the course for all three cohorts. Students' final course grades were depended on both reading participation (5\%) and correctly completing challenge activities $(5 \%)$. While all of the reading participation clicks were required to earn a perfect score, challenge activities were given a 15-question forgiveness factor (3-5\% of questions). However, the success on questions presented here encompass all attempts, and thus, do not account for the forgiveness factor.

Challenge activities are available in most sections and usually consist of 3 to 6 question levels. These question levels are scaffolded, so easier questions should precede more challenging ones $[16,25,26]$. Student must answer earlier question levels before they can proceed to the next question level. Challenge activities are primary distributed in each section with some longer questions at the end of each chapter. By integrating challenge activities into a section, new content is chunked, which is consistent with cognitive load theory $[5,27]$.

The features of one question level are summarized here to distinguish challenge activities from other online homework platforms. Rolling numbers in multiple locations as well as changing content are standard features, therefore, each question level contains tens to tens of thousands of variations. For example, a chemical reaction may change between questions in a single question level (Figure 2). In addition to being integrated into sections and having multiple levels, question levels do not limit the number of attempts before a student answers correctly. Students can seek assistance from the faculty, teaching assistants, or classmates when solving challenge activities. 
Jump to level 1

The following reaction occurs in a continuous, steady state reactor.

$1 \mathrm{~N}_{2}+3 \mathrm{H}_{2} \rightarrow 2 \mathrm{NH}_{3}$

The feed contains $7.08 \mathrm{~mol} / \mathrm{hr}$ of $\mathrm{H}_{2}$ and the conversion of $\mathrm{H}_{2}$ is

0.894. Finding the unreacted flow rate of $\mathrm{H}_{2}$ gives:

$\dot{n}_{\text {unreacted } H_{2}}=0.761$ @ $\mathrm{mol} / \mathrm{hr}$

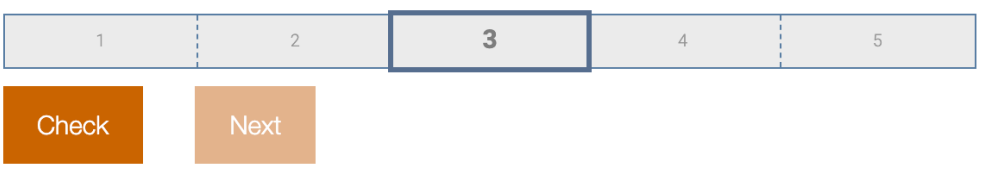

The following reaction occurs in a continuous, steady state reactor.

$8 \mathrm{Fe}+1 \mathrm{~S}_{8} \rightarrow 8 \mathrm{FeS}$

The feed contains $8.31 \mathrm{~mol} / \mathrm{hr}$ of $S_{8}$ and the conversion of $S_{8}$ is

0.329. Finding the unreacted flow rate of $S_{8}$ gives:

$\dot{n}_{\text {unreacted }, S_{8}}=0.7610 \mathrm{~mol} / \mathrm{hr}$

Figure 2. Top. One version from a question level calculating conversion. Question level is indicated as 3rd of 5 and includes Check and Next buttons used to interact with challenge activities. Bottom. Another question iteration of the same question level. The chemical reaction, feed rate, and conversion are randomly generated with each attempt.

\section{Results and discussion}

Both success and attempts on challenge activities are primary metrics to investigate the utility of online homework for the Material and Energy Balances course. Perspectives related to the course content, question type, and student success provide a broad view of these online homework questions. Content varies by chapter similar to other texts, and these topics are important for some data presented later (Table 2). 
Table 2. Chapter numbering and titles for Material and Energy Balances zyBook (January 2020).

\begin{tabular}{|l|c|}
\hline Chapters & Questions 2020 \\
\hline 1. Quantities, units, and basic calculations & 76 \\
\hline 2. Materials Balances and Question Solving & 43 \\
\hline 3. Reacting Systems & 68 \\
\hline 4. Solids, Liquids, and Gases & 41 \\
\hline 5. Multiphase systems & 46 \\
\hline 6. Energy Balances & 67 \\
\hline 7. Energy Balances For Reacting Systems & 47 \\
\hline 8. Transient Systems & 13 \\
\hline 9. Spreadsheets & 127 \\
\hline \multicolumn{2}{|r|}{ Total } \\
\hline
\end{tabular}

The percent correct for each chapter averages the success on individual question levels within each chapter (Figure 3). This success metric was recorded at the due date and is independent of the number of attempts taken before the correct answer was submitted. Some variations by cohort was observed. First, Chapters 1, 2, and 4 vary $5 \%$ or less across the three cohorts. Chapters 1, 2, and 4 cover information covered in previous introductory courses or in general chemistry, e.g., the ideal gas law in Chapter 4. Success on auto-graded questions in Chapter 3 and Chapters 5 through 8 have more fluctuation between cohorts. These chapters emphasize new concepts and question solving strategies that students may not have seen previously and in some ways define engineering thinking. For example, Chapter 5 covers multiphase systems and success on challenge activities has the most variation at $15 \%$ - ranging from 76 to $91 \%$. Spreadsheet content in Chapter 9 was introduced in 2018 with similar success by question across the two cohorts. Overall, the 2018 cohort completed the highest percentage of questions at $87 \%$ followed by $85 \%$ in 2017 , and $83 \%$ in 2019. Overall, variation across cohorts is expected, while variations across content could be explored further in a future study.

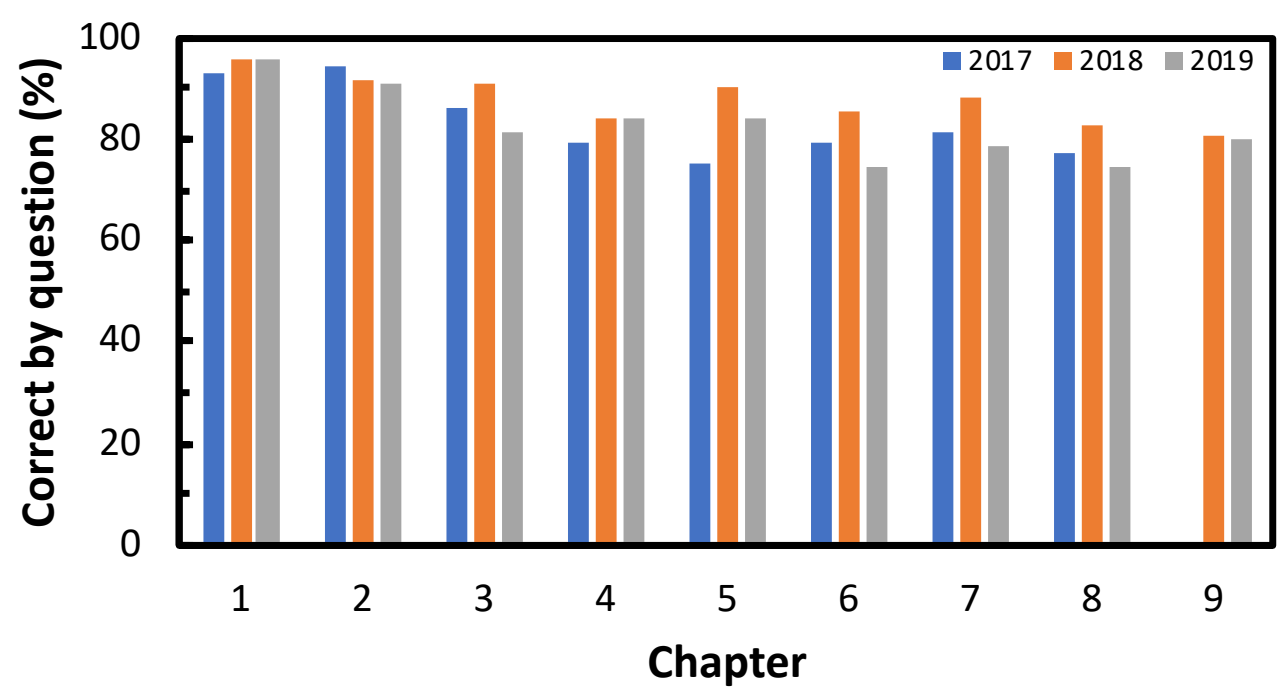

Figure 3. Correct by question (\%) for all questions in each chapter across three cohorts. 
Success using online homework varies with question type (Figure 4). Multiple choice question levels allow students to select an answer from a dropdown menu contain 3 to 5 responses. The questions still changed randomly by student and with each incorrect response. Multiple choice questions have the highest percent correct for all three cohorts at $91 \pm 3 \%$. Question levels with numerical responses were divided into a single number or multiple numbers - where all numbers need to be correct within a tolerance for the question to be consider correct. Single numeric responses led to greater success $(87 \pm 3 \%)$ than multiple numerical answers $(80 \pm 5 \%)$. These findings may not be surprising, since the effort required to correctly answer a multiple choice question (e.g., definitions or data interpretation) is normally lower than numerical response (e.g., calculations using one or more balances). Also, multiple numerical response questions are normally problems where multiple balances (e.g., atom balances) are solved simultaneously. Therefore, the decreasing student success from single to multiple numerical responses serves as a starting point for further investigation into problem difficulty.

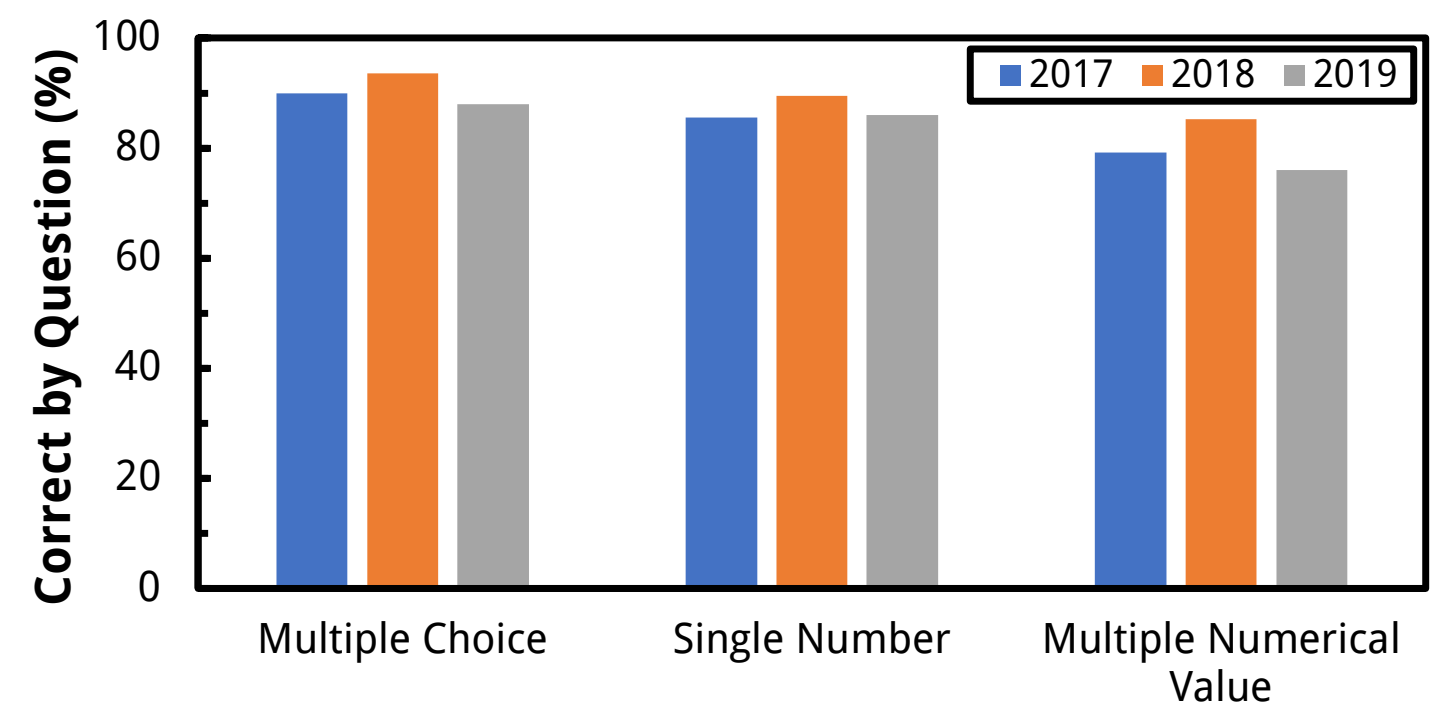

Question Type

Figure 4. Correct by question (\%) as a function of three question type across three cohorts.

Examining success on challenge activities across the three cohorts of students shows no significant variation (Figure 5). Across three cohorts, the average completion was $88 \pm 16 \%$. Mean and standard deviation allows for t-testing between the three cohorts, and each pair of cohorts shows significant statistical similarity (p-values 0.91 to 0.98 ). Alternatively, the median student correctly completed about $95 \%$ of the questions, which is measurably greater than the average. Finally, the $1^{\text {st }}$ quartile success, i.e., accounting for at least three quarters of the students, varied between 81 and $85 \%$. Therefore, presenting quartile data may be preferred to capture student success more broadly in the future. 


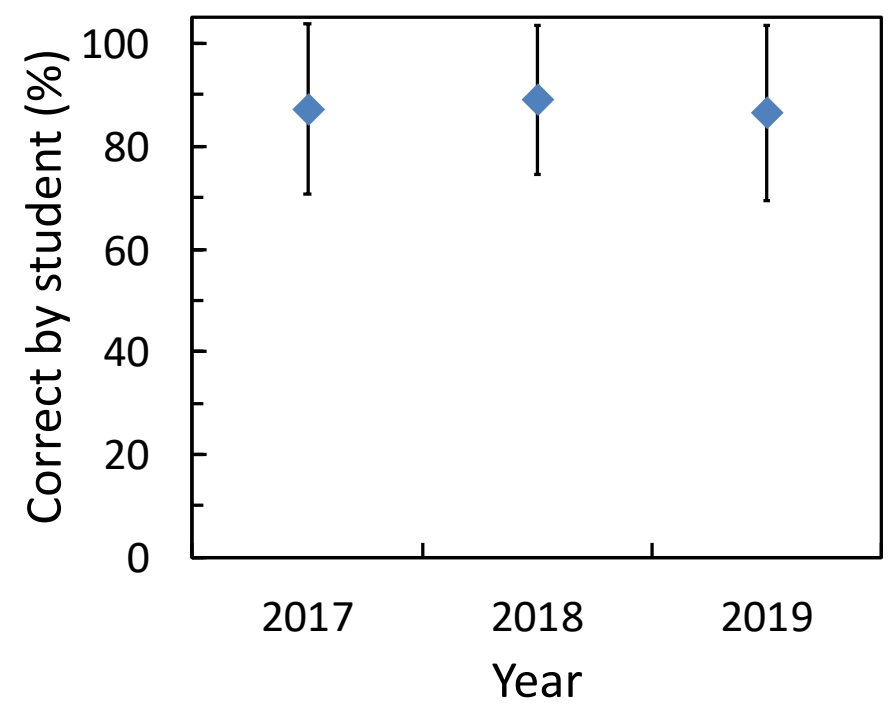

Figure 5. Correct by question (\%) across three cohorts.

Since the number of questions increased annually, the number of attempts made by each cohort also increased (Table 3). While success remained similar, as discussed above, the number of attempts before correct per question per student decreased from 2.4, 2.2, and 1.9 for 2017, 2018, and 2019 cohorts, respectively. While the decrease in average attempts before correct is quantifiable, the change is about 0.5 attempts, which will be monitored in future years.

Table 3. Questions and attempts summary by cohort and in total.

\begin{tabular}{|l|c|c|c|c|}
\hline & $\mathbf{2 0 1 7}$ & $\mathbf{2 0 1 8}$ & $\mathbf{2 0 1 9}$ & Total \\
\hline Students & 88 & 98 & 98 & 284 \\
\hline Questions & 173 & 300 & 408 & 881 \\
\hline Attempts Before Correct & 36,450 & 65,931 & 76,709 & 179,090 \\
\hline Total Attempts & 39,796 & 70,140 & 87,079 & 197,015 \\
\hline Additional Attempts & $\mathbf{3 , 3 4 9}$ & $\mathbf{4 , 2 0 9}$ & $\mathbf{1 0 , 3 7 0}$ & $\mathbf{1 7 , 9 2 5}$ \\
\hline
\end{tabular}

The increase between attempts before correct and total attempts shows that students are making more attempts after they correctly answer questions (Table 3 ). In aggregate, students made 19 practice attempts per question after correct $(19,14$, and 25, for 2017, 2018, and 2019, respectively). These practice attempts are spread over more than half of the student in many chapters. Students may be practicing more due to prompting during class or other factors. In general, thousands of additional attempts imply that auto-graded questions can serve as an active study tool before a quiz or exam. Thus, having access to questions that generate new numbers/context with each attempt seems to address the common request for more practice questions before a quiz or exam. 


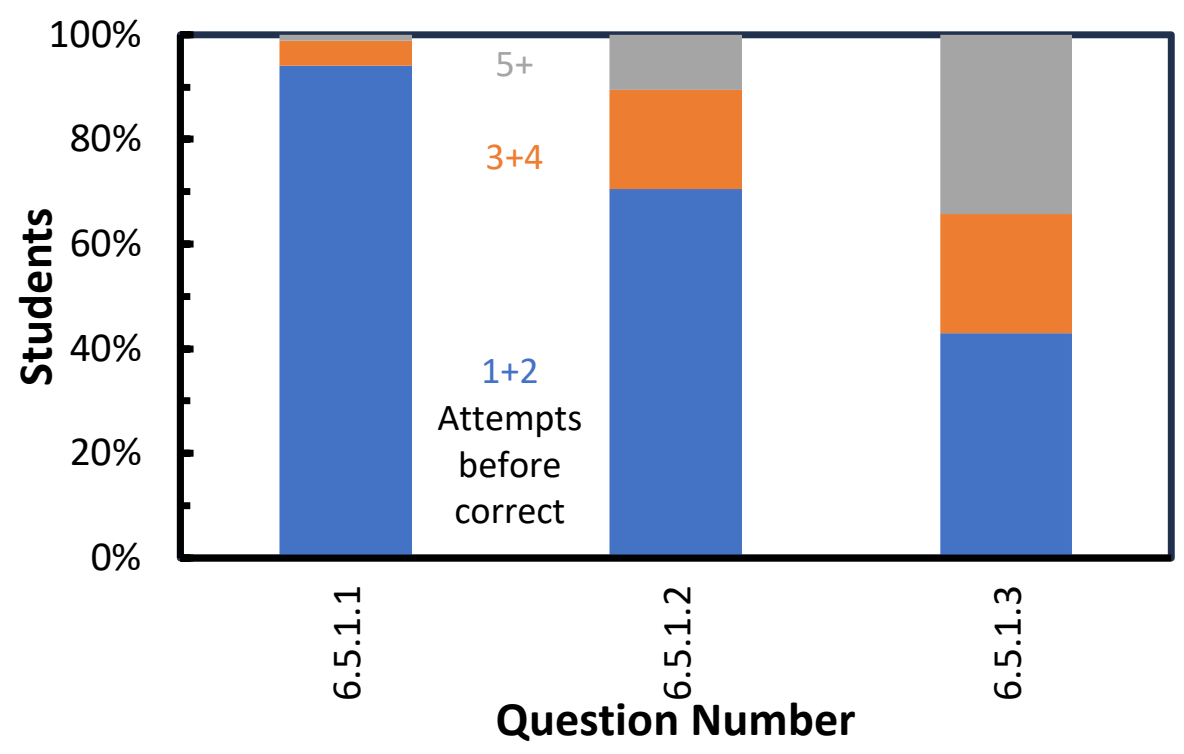

Figure 6. Histograms of the fraction of students attempts before correct across question levels for a challenge activity in Chapter 6 (6.5.1: Heat capacity calculations) for 2019 cohort.

Examining mean or median number of attempts before correct may not be most representative of the difficulty of an individual question. Histograms visualized fractions of students by attempts before correct for each question level (Figure 6). Since the questions are scaffolded, the first question (6.5.1.1) is easiest with less than $5 \%$ of students making more than 2 attempts. The fraction of students needed 3 or 4 as well as 5 or more attempts increased for the second and third question levels. By the final question level (6.5.1.3), 23\% of students made 3-4 attempts and 34\% made 5 or more attempts. Using this visualization, a difficulty metric based on percentage of students who made a high number of attempts could be built. Additionally, correlating success and attempts may allow question difficulty to be mapped. Our hypothesis is that a difficulty metric could categorize questions. Easy questions would have higher success and lower number of attempts before correct, while difficult questions would combine lower success and higher attempts. Thus, in the future, creating a difficulty metric could identify problematic questions that may need to be changed, content added, or topics requiring more class time.

\section{Conclusion}

Big data in terms of success and attempts on online homework questions were explored. Previous presentations measured median reading participation rates as high as $99 \%$ within an interactive textbook titled Material and Energy Balances zyBook. Now, allowing students multiple attempts for solving each question level led to average success of $88 \%$ over three cohorts and hundreds of questions. Success varied 5 to $15 \%$ between cohorts, and Chapters introducing new concepts led to lower success than other chapters. Multiple choice questions were easier than numerical response questions; multiple numerical responses led to 11 and 7\% lower success than multiple choice and single numerical response questions, respectively. Thousands of attempts were completed after a question was answered correctly, about 19 attempts per question, which verifies that students use these questions containing rolling numbers/content for additional practice - a widely accepted learning best practice. Finally, histograms that bin attempts before correct into categories provide a visual framework to identify when a significant fraction of students are 
struggling with a question level. Future work will attempt to correlate success and attempts to identify difficult questions and improve student success using the interactive textbook, which hopefully would translate to improved outcomes in the course also.

\section{Acknowledgments}

The authors thank recent contributions from Katherine Roach and Alex Edgcomb, and numerous teaching assistants. This work was completed within the framework of University Toledo IRB protocol 201808.

\section{Disclaimer}

One of the authors may receive royalties from sales of the zyBook detailed in this paper.

\section{Bibliography}

[1] B. Poulson. "Big Data in Data Science Foundations: Fundamentals." https://www.lynda.com/IT-tutorials/Big-data/2805908/2232722-4.html (accessed January, 2020).

[2] S. Stephens-Davidowitz and S. Pinker, Everybody lies: big data, new data, and what the internet can tell Us about who we really are. HarperCollins New York, 2017.

[3] A. Edgcomb and F. Vahid, "Effectiveness of Online Textbooks vs. Interactive WebNative Content," in ASEE Annual Conference, Indianapolis, IN, 2014, doi: https://peer.asee.org/20351.

[4] A. Edgcomb, F. Vahid, R. Lysecky, A. Knoesen, R. Amirtharajah, and M. L. Dorf, "Student Performance Improvement using Interactive Textbooks: A Three- University Cross-Semester Analysis," in ASEE Annual Meeting, Seattle, WA, 2015, doi: https://doi.org/10.18260/p.24760.

[5] M. T. Chi, "Active-constructive-interactive: a conceptual framework for differentiating learning activities," Topics in Cognitive Science, vol. 1, no. 1, pp. 73-105, Jan 2009, doi: https://doi.org/10.1111/j.1756-8765.2008.01005.x.

[6] A. W. Chickering and Z. F. Gamson, "Seven Principles for Good Practice in Undergraduate Education," AAHE Bulletin, pp. 1-7, 1987.

[7] S. Freeman et al., "Active learning increases student performance in science, engineering, and mathematics," Proceedings of the National Academy of Sciences, vol. 111, no. 23, pp. 8410-8415, 2014, doi: https://10.1073/pnas.1319030111.

[8] R. M. Felder and R. Brent, Teaching and Learning STEM: A Practical Guide. San Francisco, CA: Jossey-Bass, 2016.

[9] National Academy of Engineering, Educating the engineer of 2020: adapting engineering education in the new century. National Academies Press, 2005.

[10] C. C. Martin, W. C. Newstetter, and J. M. Le Doux, "Inclusion requires a comprehensive understanding of justice," Journal of Engineering Education, vol. 108, no. 4, pp. 453458, 2019, doi: https://10.1002/jee.20296.

[11] M. W. Liberatore, "High textbook reading rates when using an interactive textbook for a Material and Energy Balances course," Chemical Engineering Education, vol. 51, no. 3, pp. 109-118, 2017, doi: https://journals.flvc.org/cee/article/view/104416. 
[12] M. W. Liberatore, "Reading analytics and student performance when using an interactive textbook for a material and energy balances course," in ASEE Annual Conference \& Exposition, Columbus, OH, 2017, pp. 1-13, doi: https://peer.asee.org/28780.

[13] M. W. Liberatore and K. Roach, "Quantifying Self-guided Repetition Within an Interactive Textbook for a Material and Energy Balances Course," in ASEE Annual Meeting, Salt Lake City, UT, 2018, pp. 1-12, doi: https://peer.asee.org/30912.

[14] M. W. Liberatore and K. Chapman, "Reading Anytime: Do Students Complete Missed Readings After the Due Date When Using an Interactive Textbook for Material and Energy Balances?," in ASEE Annual Conference, Tampa, FL, 2019, pp. 1-10, doi: https://peer.asee.org/33224.

[15] M. W. Liberatore, K. E. Chapman, and K. M. Roach, "Significant reading participation across multiple cohorts before and after the due date when using an interactive textbook," Computer Applications in Engineering Education, vol. 28, no. 2, pp. 444-453, 2020, doi: https://doi.org/10.1002/cae.22210.

[16] J. M. Lang, Small Teaching: Everyday Lessons from the Science of Learning. John Wiley \& Sons, 2016.

[17] McGraw-Hill. "Aleks: Assessment and LEarning in Knowledge Spaces " https://www.aleks.com/ (accessed January, 2020).

[18] G. Kortemeyer, E. Kashy, W. Benenson, and W. Bauer, "Experiences using the opensource learning content management and assessment system LON-CAPA in introductory physics courses," American Journal of Physics, vol. 76, no. 4, p. 438, 2008, doi: 10.1119/1.2835046.

[19] E. McGroarty et al., "Supplementing introductory biology with on-line curriculum*," Biochemistry and Molecular Biology Education, vol. 32, no. 1, pp. 20-6, Jan 2004, doi: https://doi.org/10.1002/bmb.2004.494032010312.

[20] P. S. Steif and A. Dollar, "Study of Usage Patterns and Learning Gains in a Web-based Interactive Static Course," (in English), Journal of Engineering Education, vol. 98, no. 4, pp. 321-333, Oct 2009, doi: https://doi.org/10.1002/j.2168-9830.2009.tb01030.x.

[21] M. W. Liberatore, "Improved student achievement using personalized online homework for a course in material and energy balances," Chemical Engineering Education, vol. 45, no. 3, pp. 184-190, 2011.

[22] J. Medina, Brain Rules 12 Principles for Surviving and Thriving at Work, Home, and School. Pear Press, 2008.

[23] D. L. Silverstein, L. G. Bullard, and M. A. Vigeant, "How we teach: Material and Energy Balances," in ASEE Annual Meeting, San Antonio, TX, 2012, p. 3583.

[24] M. W. Liberatore, "Active Learning and Just-in-time Teaching In a Material and Energy Balances Course," Chemical Engineering Education, vol. 47, no. 3, pp. 154-160, 2013, doi: https://journals.flvc.org/cee/article/view/114520.

[25] B. J. S. Barron et al., "Doing with understanding: Lessons from research on problem- and project-based learning," (in English), Journal of the Learning Sciences, vol. 7, no. 3-4, pp. 271-311, 1998, doi: https://doi.org/10.1080/10508406.1998.9672056.

[26] N. K. Lape, "Tiered Scaffolding of Problem-Based Learning Techniques in a Thermodynamics Course," in ASEE Annual Conference, 2011: American Society for Engineering Education. 
[27] E. D. Sloan and C. Norrgran, "A neuroscience perspective on learning," Chemical Engineering Education, vol. 50, no. 1, pp. 29-37, 2016, doi:

https://journals.flvc.org/cee/article/view/87714. 